\title{
Ion-Source Modeling and Improved Performance of the CAMS High-Intensity Cs-Sputter Ion Source
}

\author{
T.A. Brown, M.L. Roberts and J.R. Southon
}

This article was submitted to

$8^{\text {th }}$ International Conference on Accelerator Mass Spectrometry Vienna, Austria

September 6-10, 1999

U.S. Department of Energy

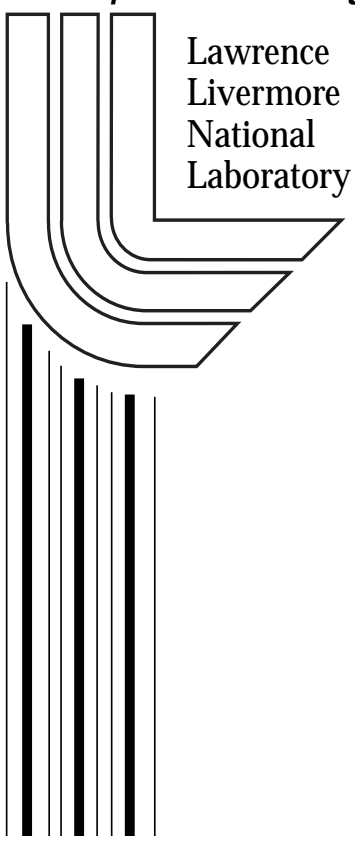

October 22, 1999 


\section{DISCLAIMER}

This document was prepared as an account of work sponsored by an agency of the United States Government. Neither the United States Government nor the University of California nor any of their employees, makes any warranty, express or implied, or assumes any legal liability or responsibility for the accuracy, completeness, or usefulness of any information, apparatus, product, or process disclosed, or represents that its use would not infringe privately owned rights. Reference herein to any specific commercial product, process, or service by trade name, trademark, manufacturer, or otherwise, does not necessarily constitute or imply its endorsement, recommendation, or favoring by the United States Government or the University of California. The views and opinions of authors expressed herein do not necessarily state or reflect those of the United States Government or the University of California, and shall not be used for advertising or product endorsement purposes.

This is a preprint of a paper intended for publication in a journal or proceedings. Since changes may be made before publication, this preprint is made available with the understanding that it will not be cited or reproduced without the permission of the author.

This report has been reproduced directly from the best available copy.

Available to DOE and DOE contractors from the

Office of Scientific and Technical Information

P.O. Box 62, Oak Ridge, TN 37831

Prices available from (423) 576-8401

http:/ / apollo.osti.gov/bridge/

Available to the public from the National Technical Information Service

U.S. Department of Commerce 5285 Port Royal Rd., Springfield, VA 22161 http://www.ntis.gov/

OR

Lawrence Livermore National Laboratory Technical Information Department's Digital Library http://www.llnl.gov/tid/Library.html 
Manuscript for submission to the Journal

Nuclear Instrument and Methods in Physics Research B

as a contribution to the

Proceeding of the Eighth International Conference on Accelerator Mass Spectrometry

Vienna, Austria, September 6-10, 1999.

Authors:

T. A. Brown, M. L. Roberts, and J. R. Southon

Center for Accelerator Mass Spectrometry

LLNL, P.O. Box 808, Livermore, CA 94551, U.S.A.

Title:

Ion-source modeling and improved performance of the CAMS high-intensity Cs-sputter ion source. 


\title{
Ion-source modeling and improved performance of the CAMS high-intensity Cs-sputter ion source.
}

\author{
T. A. Brown, M. L. Roberts, and J. R. Southon \\ Center for Accelerator Mass Spectrometry, LLNL, P.O. Box 808, Livermore, CA 94551, U.S.A.
}

\begin{abstract}
The interior of the high-intensity Cs-sputter source used in routine operations at the Center for Accelerator Mass Spectrometry (CAMS) has been computer modeled using the program NEDLab, with the aim of improving negative ion output. Space charge effects on ion trajectories within the source were modeled through a successive iteration process involving the calculation of ion trajectories through Poisson-equation-determined electric fields, followed by calculation of modified electric fields incorporating the charge distribution from the previously calculated ion trajectories. The program has several additional features that are useful in ion source modeling: 1) averaging of space charge distributions over successive iterations to suppress instabilities, 2) Child's Law modeling of space charge limited ion emission from surfaces, and 3) emission of particular ion groups with a thermal energy distribution and at randomized angles.

The results of the modeling effort indicated that significant modification of the interior geometry of the source would double $\mathrm{Cs}^{+}$ion production from our spherical ionizer and produce a significant increase in negative ion output from the source. The results of the implementation of the new geometry were found to be consistent with the model results.
\end{abstract}

Keywords: Ion sources, Charged-particle beam sources, Mass spectrometry; Computer codes. Corresponding Author:

T. A. Brown, CAMS L-397, LLNL, P.O. Box 808, Livermore, CA 94551, U.S.A fax: 925-423-7884, telephone: 925-423-8507, email: tabrown@1lnl.gov 


\section{Introduction}

The ion sources currently used at CAMS are based on a General Ionex Corporation 846 Cs sputter ion source purchased in 1989. Since the time of purchase, considerable effort has been expended in initial work to make the source operational [1], and subsequently, in modifications to improve the performance of the source to meet the high-output, high-reliability demands of day-to-day AMS operations [2]. These efforts, which have been described by Southon and Roberts [3], have resulted in an ion source that routinely produces negative ion currents of 200$250 \mu \mathrm{A} \mathrm{C}^{-}, 15-20 \mu \mathrm{A} \mathrm{BeO}^{-}$, or $0.5-1.0 \mu \mathrm{A} \mathrm{CaF}_{3}^{-}$, and that will operate essentially continuously at such output levels for 2-3 weeks before needing maintenance.

In an effort to further improve the performance of our ion sources, the interior of our highintensity Cs-sputter source has been computer modeled using NEDLab, a grid-point mesh program. The model of our source was constructed on a 600-by-300 grid, and the cylindrically symmetric electrode structures of the source were input via a PICT file derived directly from CAD drawings used in constructing the actual ion source. Minor adjustments were made to the model's electrodes based on measurements of a routinely used source.

The NEDLab program has several features that are useful in modeling high-intensity Cssputter sources. First, space-charge effects are incorporated in the calculations through an automated ion-trajectories/Poisson-electric-fields successive-iteration process. Second, NEDLab allows ion emission from surfaces to be Child's Law constrained to account for limitations on ion emission due to the space charge of previously emitted ions in the near-surface region. Third, the program allows the averaging of charge distributions over successive iterations to aid in the suppression of model oscillations. These typically arise when the presence of ions in a particular region during one iteration causes significant modifications of the electric fields such that the ions are not present in that region during the next iteration; but, their "non-presence" in the second iteration causes the electric fields to revert to their previous state and the ions are once again present in the region during the following (third) iteration. The option to average charge state distributions over several iterations appears to significantly reduce model sensitivity to conditions that could cause the onset of such oscillatory behavior. Fourth, the program allows 
the emission energy of particular ion groups from a surface to be described by a thermal energy distribution, with individual ion energies being sampled randomly from within that energy distribution. And finally, NEDLab allows surface-emission ions to be emitted at a random angle to the emitting surface.

The model encompassed the source's ionizer/target region and contained the relevant parts of four electrodes: 1) the spherical ionizer, 2) the ionizer shroud, 3) the sample target (cathode), and 4) the target shield (immersion lens). The prescribed potentials for these electrodes were set to reflect nominal ion source operating conditions; i.e., the sample target and target shield were set at $-39 \mathrm{kV}$ and the spherical ionizer and ionizer shroud at $-30 \mathrm{kV} . \mathrm{Cs}^{+}$emission from the ionizer surface was Child's Law constrained, and the $\mathrm{Cs}^{+}$ions were emitted with a thermal energy distribution having a characteristic energy of $0.15 \mathrm{eV}$ and at randomized angles to the local surface of the ionizer. The emission of ${ }^{12} \mathrm{C}^{-}$and ${ }^{12} \mathrm{C}_{2}^{-}$ions was limited to the normally sputtered sample area of the target, and the carbon ions were emitted with a $15 \mathrm{eV}$ thermal energy distribution at randomized angles to the local target surface. The ${ }^{12} \mathrm{C}_{2}{ }^{-}$current was set to be $1 / 3$ that of the ${ }^{12} \mathrm{C}^{-}$ions, based on previous in-house measurements of mass 12 and mass 24 negative carbon ion production from the source.

While the Child's Law constraint on $\mathrm{Cs}^{+}$emission determined the intensity of emission of these positive ions automatically, NEDLab's Child's Law algorithms led to computational failures when applied to the emission of carbon ions from the sample target. In order to estimate the $\mathrm{C}^{-}$current that would be produced with a given geometry, several model runs were performed for each geometry. $\mathrm{C}^{-}$emission was increased from run to run until the space charge from $\mathrm{C}^{-}$ emission was sufficient to overwhelm the potential gradient at the sample surface and $\mathrm{C}^{-}$output oscillated under iterative cycling.

\section{Model of Present Ion Source Geometry}

The model results obtained for our present ion source geometry are shown in Figure 1. The modeled $\mathrm{Cs}^{+}$trajectories (Fig. 1a) reproduce almost all observed features of the focussing of the $\mathrm{Cs}^{+}$ions emitted from the ionizer surface onto the sample target during normal operation of our 
present source. In particular, the focussing of the $\mathrm{Cs}^{+}$ions to a spot well within the $1.3 \mathrm{~mm}$ diameter sample well agrees with the sputtering patterns observed on the surface of expended targets. In addition, the model's pattern of poorly focussed $\mathrm{Cs}^{+}$ions emitted from the inner corner region of the ionizer is observed as a "sputter halo" on expended targets. Integration of modeled $\mathrm{Cs}^{+}$emission over the ionizer surface provides a $\mathrm{Cs}^{+}$current estimate of $\sim 1.3 \mathrm{~mA}$ under nominal operating conditions, which is consistent with measured currents.

(Figure 1. Here)

The modeled negative ion trajectories (Fig. 1b) are consistent with observed features during normal operations. The lack of beam markings in the region surrounding the central hole of used ionizers indicates that the sputtered negative ion beams are extracted cleanly through the central hole of the ionizer, as in the model. Integration of modeled ${ }^{12} \mathrm{C}^{-}$emission over the sample surface provides a ${ }^{12} \mathrm{C}^{-}$current estimate of $\sim 200 \mu \mathrm{A}$, comparable with observed $200-250 \mu \mathrm{A}^{12} \mathrm{C}^{-}$ outputs. Interestingly, the modeled carbon ion trajectories show that the $\mathrm{C}^{-}$ion beam forms a waist about halfway between the target and the ionizer, and is diverging as it exits the source through the central ionizer hole. In addition, there does not appear to be a significant difference between the ${ }^{12} \mathrm{C}^{-}$and ${ }^{12} \mathrm{C}_{2}^{-}$trajectories, suggesting that mass dependent effects during sputteremission do not translate into significant emittance differences between the two ion species as they are transported out of the ion source.

\section{Modified Ion Source Geometry Model}

The model results obtained for the modified ion source geometry are shown in Fig. 2. This geometry reflects the end-product of a series of models that investigated the impact of various possible electrode modifications. These "modification" models were run under the same conditions as the model of our present source, with the following two exceptions: 1) various modifications were made to the ionizer shroud, sample target, and target shield geometries, and 2) the target shield potential was varied. Over the course of series, the target shield was 
extended towards the spherical ionizer to increase the electric field near the surface of the ionizer. Significant alterations to the geometries of the extended target shield and the ionizer shroud were then required to maintain focussing of the $\mathrm{Cs}^{+}$ions on the sample target. The target shield was further modified through the addition of an "extraction plane" extending inward toward the central axis of the source, parallel to the surface of the target. In conjunction with the "extraction plane", the sample target was modified to remove the conical bowl surrounding the sample well and to bring the sample surface flush with the now flat surface of the target. The target shield's extraction plane was placed at an appropriate distance from the target surface to allow a potential difference across the target/target-shield gap to introduce a significant electric field near the sample surface.

(Figure 2. Here)

The modeled $\mathrm{Cs}^{+}$trajectories in the modified geometry ion source (Fig. 2a) indicate that the new ionizer shroud and target shield maintained the focussing of the emitted $\mathrm{Cs}^{+}$ions onto the $1.3 \mathrm{~mm}$ diameter sample position. However, the focussed $\mathrm{Cs}^{+}$ions now covered the entire sample diameter rather than the smaller diameter focus achieved in the present source model (cf. Fig. 1a). The $\mathrm{Cs}^{+}$focussing in the modified model appears to be more complex than for the present geometry model in that the ions from the outer edges of the ionizer are significantly overfocussed and cross over the central axis before reaching the sample surface. Comparison of the positions of the equipotential contours in the modified and present geometry models shows that the extension of the target shield towards the ionizer has increased the electric field increase near the ionizer surface. Integration of $\mathrm{Cs}^{+}$emission over the ionizer surface provides a $\mathrm{Cs}^{+}$current estimate of $\sim 2.4 \mathrm{~mA}$ from the ionizer in the modified geometry source, almost double the $\mathrm{Cs}^{+}$ current of the present source model.

The modeled ${ }^{12} \mathrm{C}^{-}$and ${ }^{12} \mathrm{C}_{2}^{-}$trajectories in the modified geometry source (Fig. 2b) indicate that the sputtered negative ion beams will be extracted cleanly through the central hole of the ionizer. Integration of modeled ${ }^{12} \mathrm{C}^{-}$emission over the sample surface provides a ${ }^{12} \mathrm{C}^{-}$current 
estimate of $\sim 400 \mu \mathrm{A}$, double the ${ }^{12} \mathrm{C}^{-}$current of the present geometry model. In the modified geometry model the carbon ion trajectories do not appear to form a waist between the target shield and ionizer, and appear to be diverging significantly less when passing through the central ionizer hole (cf., Fig. 1b). The calculated emittance of the ${ }^{12} \mathrm{C}^{-}$ion beam from the modified geometry model is $25 \%$ smaller than that of the present geometry model. As for the present geometry model, the ${ }^{12} \mathrm{C}^{-}$and ${ }^{12} \mathrm{C}_{2}^{-}$ion beam emittances do not appear to differ significantly.

\section{Initial Test Results}

Based on the results obtained from the modified geometry model, new versions of the target shield and ionizer shroud were designed and machined from stainless steel. An electricallyinsulating alumina support for the modified target shield was constructed, and an extra power supply and associated controls were installed, to allow the target shield potential to differ from that of the target.

Initial tests of the modified ion source geometry were conducted using graphitized Oxalic Acid Standard (OX1) samples that are routinely used in our natural ${ }^{14} \mathrm{C}$ AMS measurements. These samples were mounted in targets that had been modified to remove the conical-bowl/deepwell configuration of our routine targets with the modified flat-top/flush-mount target configuration of the modified geometry model.

During the initial stages of our tests, while we were optimizing operational conditions, ${ }^{12} \mathrm{C}^{-}$ output levels $\geq 390 \mu \mathrm{A}$ were observed from several samples. After establishing operational conditions, a series of samples were measured using our routine natural ${ }^{14} \mathrm{C}$ AMS measurement scheme. All of these $\mathrm{OX} 1$ samples produced ${ }^{13} \mathrm{C}^{4+}$ currents corresponding to $\geq 360 \mu \mathrm{A}{ }^{12} \mathrm{C}^{-}$ currents and ${ }^{14} \mathrm{C}$ count rates were consistently around $1.3 \mathrm{kHz}$, allowing $1 \%$ counting statistics measurements to be made in about $8 \mathrm{~s}$. As is the case during our routine natural ${ }^{14} \mathrm{C}$ AMS measurements, the ${ }^{14} \mathrm{C} /{ }^{13} \mathrm{C}$ ratios obtained using the modified geometry source did not show significant drift over the course of repeated measurements of individual samples (during which the samples were sputtered away and the ion currents eventually dropped to less than 5\% of their initial $\geq 360 \mu \mathrm{A}$ values). 


\section{Conclusions}

The grid-point mesh program NEDLab has been used to model the interior of the highintensity Cs-sputter source used in routine operations at CAMS. Comparison of results from a model based on the present electrode geometry to the observed performance of the source showed that the $\mathrm{Cs}^{+}$and $\mathrm{C}^{-}$ion trajectories within the modeled source, and the model's estimates of the $\mathrm{Cs}^{+}$and ${ }^{12} \mathrm{C}^{-}$ion currents, were consistent with observed source performance.

Subsequent modeling investigations led to the development of a modified electrode geometry for the interior of our high-intensity Cs-sputter source. The geometries of the ionizer shroud, target shield, and target were modified to increase the electric fields near the spherical ionizer surface and the sample surface. These modifications resulted in a model-predicted factor of two increase in $\mathrm{Cs}^{+}$emission from the ionizer, and a doubling of the $\mathrm{C}^{-}$ion current produced by the ion source.

Initial tests of a developmental prototype version of the modified ion source geometry demonstrated stable operation of this geometry in the ion source, and the test results were consistent with the predictions of the modified geometry model. ${ }^{12} \mathrm{C}^{-}$current production with this modified geometry reached $\geq 390 \mu \mathrm{A}$ for several samples, and the observed $\mathrm{Cs}^{+}$sputtering pattern on the surface of test targets was consistent with the predicted $\mathrm{Cs}^{+}$focussing. The ${ }^{14} \mathrm{C} /{ }^{13} \mathrm{C}$ data obtained during these tests showed no evidence of isotope ratio drift due to changes in sample surface geometry caused by $\mathrm{Cs}^{+}$sputtering during the measurement of the samples. The results obtained in these initial tests demonstrated the utility of NEDLab-based modeling in the development and execution of modifications to high-intensity Cs sputter ion sources, and have provided the basis for further study of performance enhancing modifications to our ion sources.

\section{Acknowledgements}

This work was performed under the auspices of the U.S. Department of Energy at the Lawrence Livermore National Laboratory under contract W-7405-Eng-48. NedLab ${ }^{\mathrm{TM}}$ is available from AccelSoft Inc., www.ghga.com/accelsoft. 


\section{References}

[1] I.D. Proctor, J.R. Southon, M.L. Roberts, J.C. Davis, D.W. Heikkinen, T.L. Moore, J.L. Garibaldi, and T.A. Zimmerman, Nucl. Instr. And Methods B 52 (1990) 334.

[2] M.L. Roberts, P.J. Norman, J.L. Garibaldi, and R.S. Hornady, Nucl. Instr. And Methods B 92 (1994) 111.

[3] J.R. Southon, and M.L. Roberts, “Ten years of sourcery at CAMS/LLNL: evolution of a Cs ion source," these proceedings (1999). 


\section{Figure Captions}

Figure 1. NEDLab model results obtained for the present ion source electrode geometry. The central axis of the cylindrically symmetric ion source lies along the top edge of the model. The electrodes of the source model (matt grey) are labeled and their nominal operating potentials are indicated. Equipotential contours between the electrodes (black lines) are drawn at $225 \mathrm{~V}$ intervals. (a) Trajectories of $\mathrm{Cs}^{+}$ions emitted from the spherical ionizer surface and focused on the target by the potential gradients within the source. Different shades of grey have been used for the $\mathrm{Cs}^{+}$ions emitted from three areas of the ionizer surface to clarify the trajectories followed by particular $\mathrm{Cs}^{+}$ions. (b) Trajectories of $\mathrm{C}^{-}$sputter-emitted from the bottom of the sample well in the target and transported out of the source through the central hole of the ionizer. The trajectories of ${ }^{12} \mathrm{C}^{-}$and ${ }^{12} \mathrm{C}_{2}^{-}$ions are displayed, but are essentially identical and cannot be differentiated in the figure.

Figure 2. NEDLab model results obtained for the modified ion source electrode geometry. The central axis of the cylindrically symmetric ion source lies along the top edge of the model. The electrodes of the source model (matt grey) are labeled and their nominal operating potentials are indicated. The "extraction plane" of the modified target shield discussed in the text is shown as the vertical extension toward the central axis of the source lying just in front of the target surface. Equipotential contours between the electrodes (black lines) are drawn at $225 \mathrm{~V}$ intervals. (a) Trajectories of $\mathrm{Cs}^{+}$ions emitted from the spherical ionizer surface and focused on the target by the potential gradients within the source. Different shades of grey have been used for the $\mathrm{Cs}^{+}$ ions emitted from three areas of the ionizer surface to clarify the trajectories followed by particular $\mathrm{Cs}^{+}$ions. (b) Trajectories of $\mathrm{C}^{-}$sputter-emitted from the sample position on the target surface and transported out of the source through the central hole of the ionizer. The trajectories of ${ }^{12} \mathrm{C}^{-}$and ${ }^{12} \mathrm{C}_{2}^{-}$ions are displayed, but are essentially identical and cannot be differentiated in the figure. 


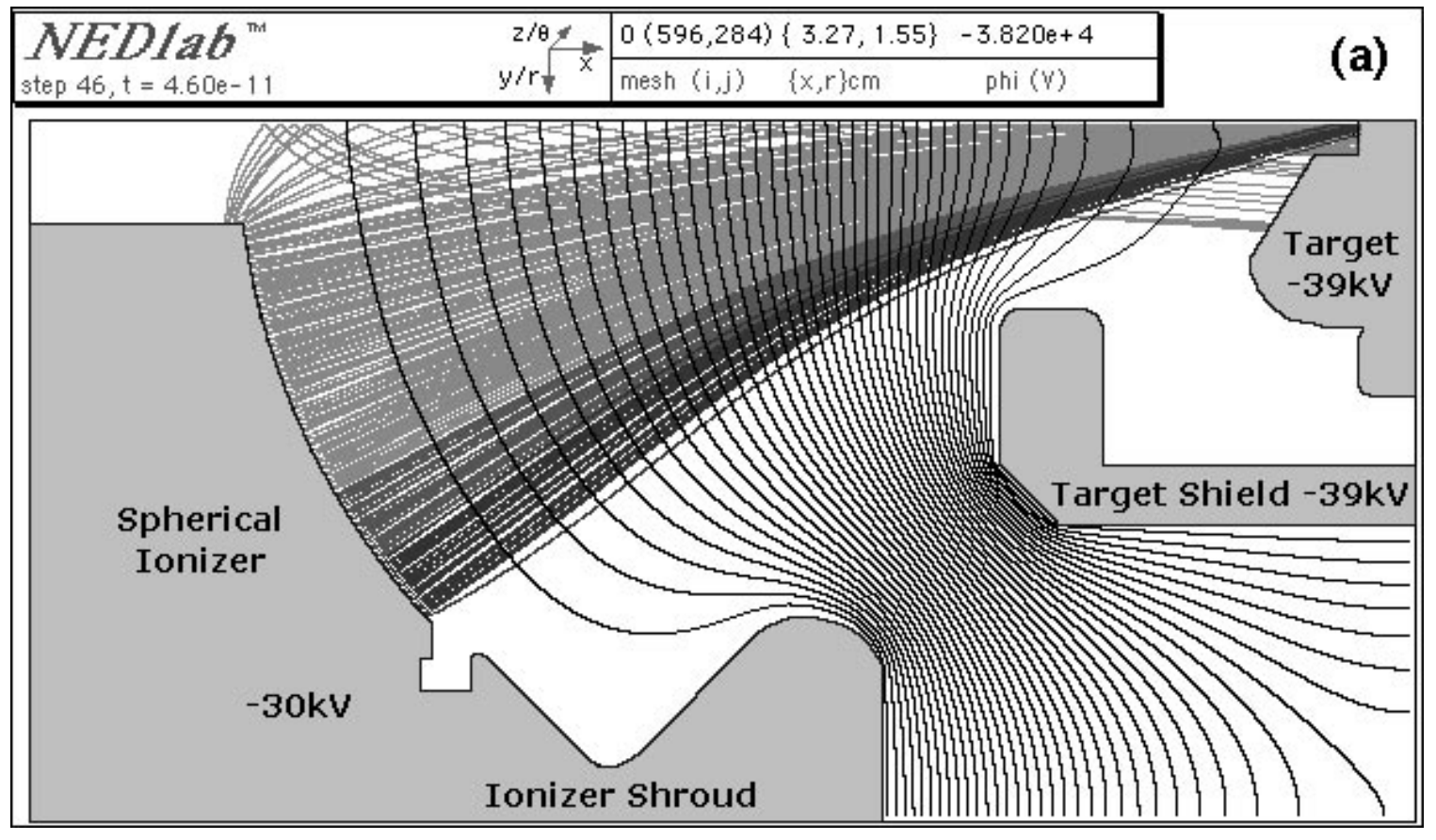




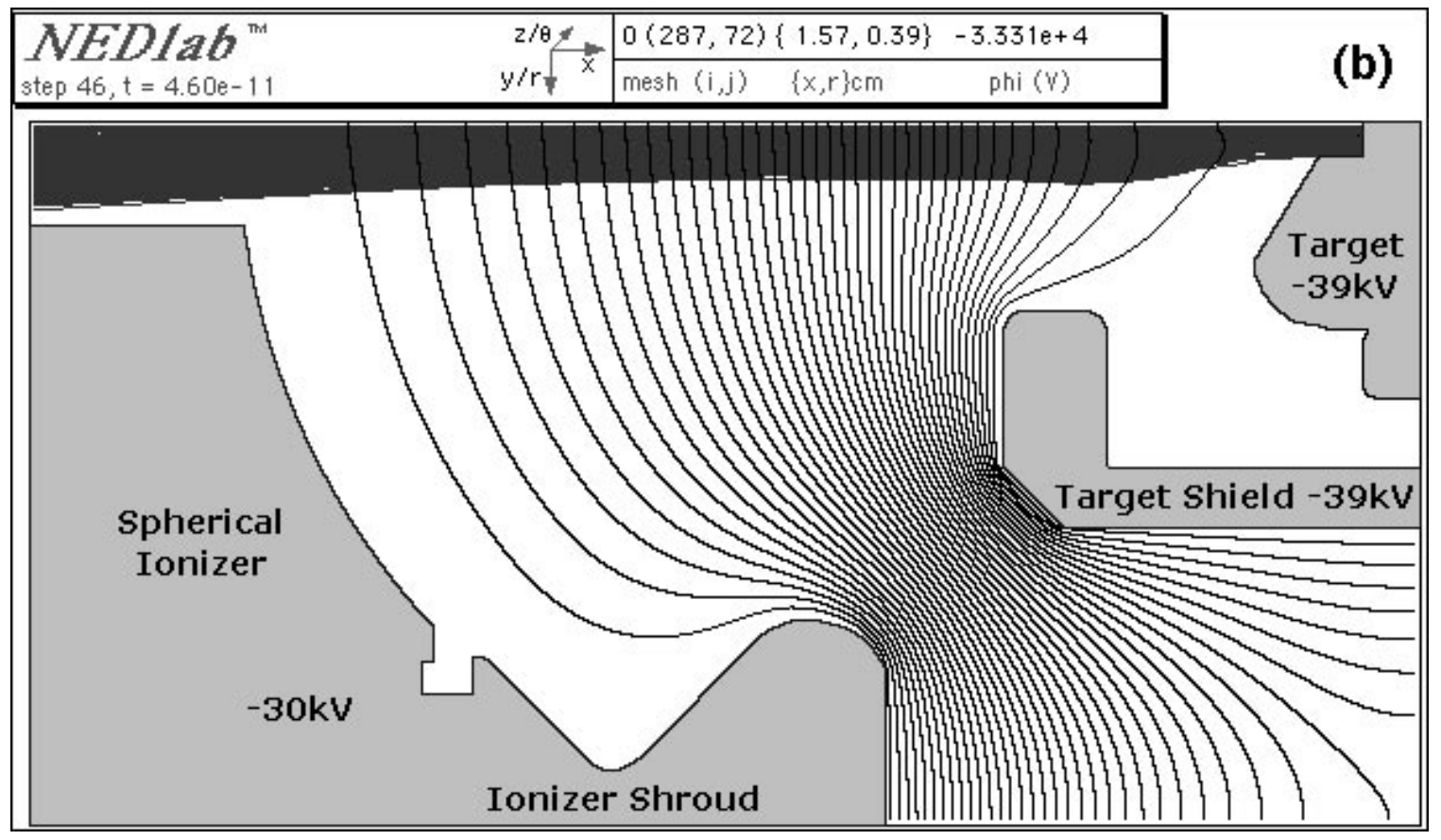




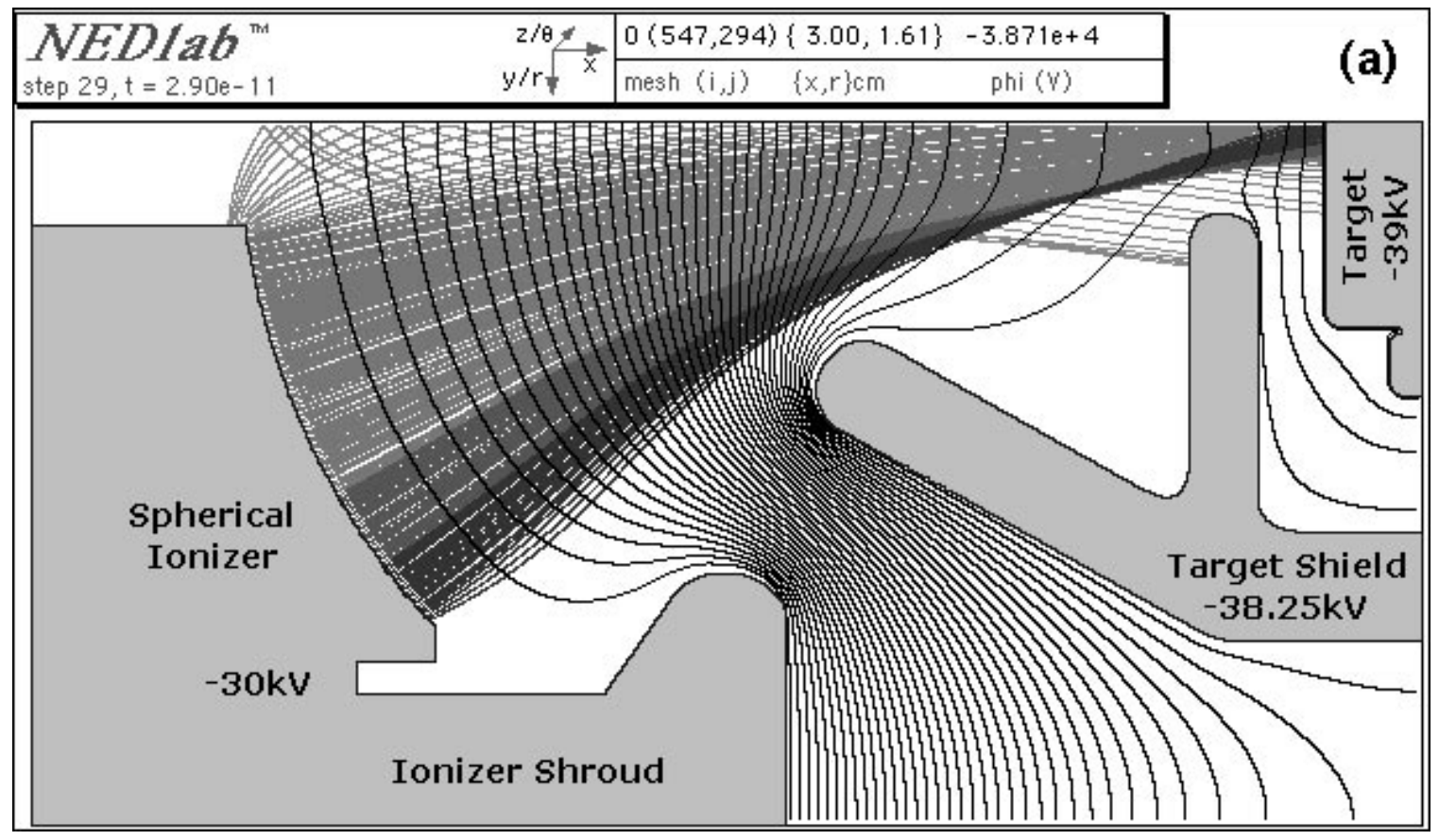




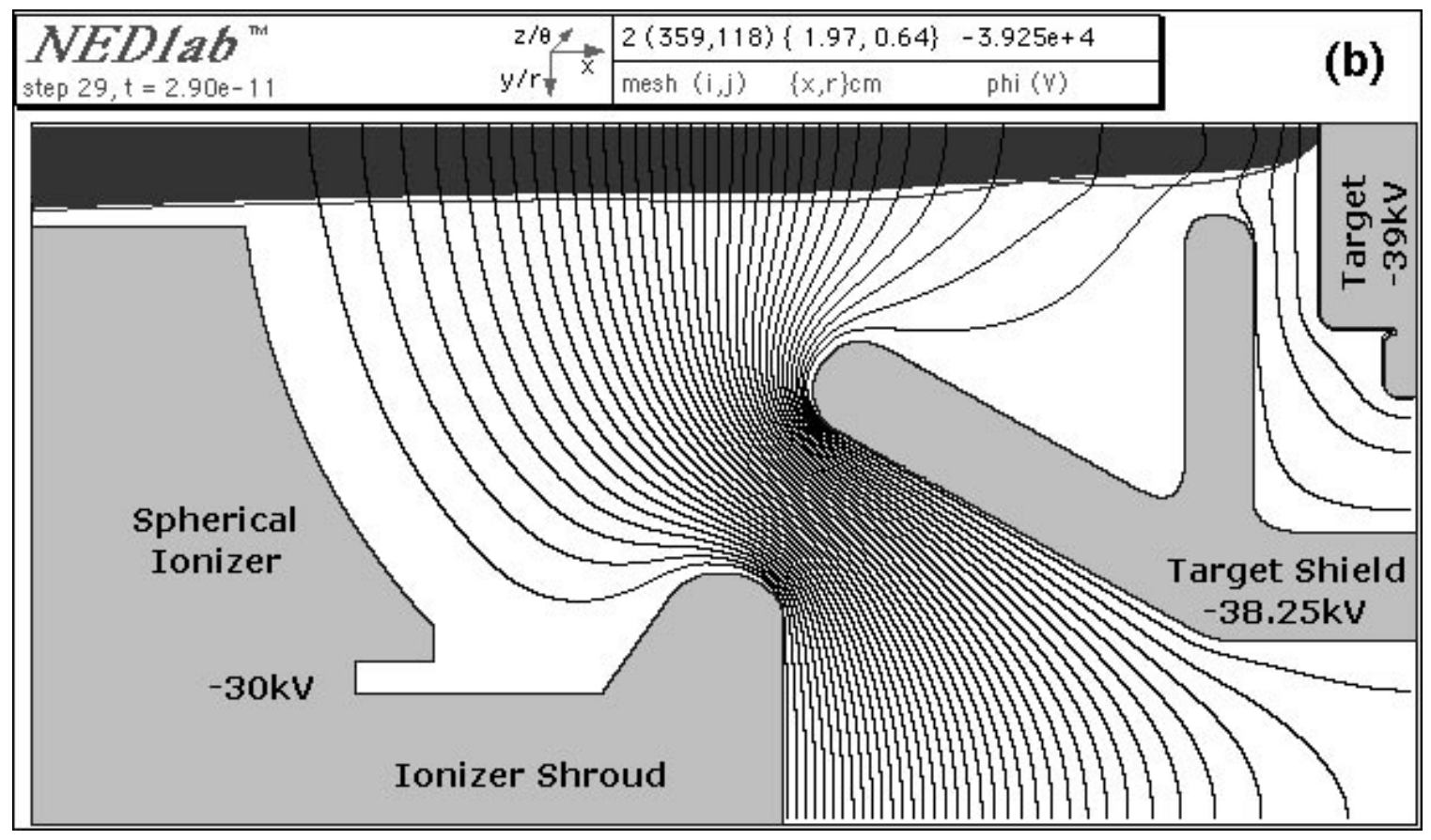

